\title{
Reversible cerebral vasoconstriction syndrome: case report
}

\author{
Oğuzhan Öz $\cdot$ Şeref Demirkaya $\cdot$ Semai Bek $\cdot$ \\ Erdal Eroğlu · Ümit Hıdır Ulaş · Zeki Odabaşı
}

Received: 6 February 2009/ Accepted: 25 March 2009/Published online: 16 April 2009

(c) Springer-Verlag 2009

\begin{abstract}
A 28-year-old woman had thunderclap headache (TCH), after 7 days she had left hemiparesis. She had a history of oral contraceptive and citalopram medications. Brain magnetic resonance (MR) angiography demonstrated multiple stenotic segments. Digital subtraction angiography (DSA) showed multiple segments of narrowing in vessel calibre. Two probable diagnoses performed; primary angiitis of the central nervous system and reversible cerebral vasoconstriction syndrome (RCVS). Because of clinical characteristics and normal cerebrospinal fluid findings she was set on medication for probable RCVS. Followup MR angiography after 4 weeks and DSA after 7 weeks demonstrated improvement in vessel calibre. Thus, diagnosis RCVS was established. Diagnosis and management of TCH contain many potential difficulties. Clinicians should consider the imaging of cerebral arteries, even if computed tomography scan and lumbar puncture are normal in TCH. Potential precipitating factors and triggers should also be known and avoided.
\end{abstract}

Keywords Reversible cerebral vasoconstriction syndrome . Thunderclap headache $\cdot$ Digital subtraction angiography $\cdot$ Selective-serotonin reuptake inhibitors

This case was presented as a poster at the European Headache and Migraine Trust International Congress (EHMTIC), 4-7th September 2008, London, UK entitled "The Call-Fleming Syndrome: case report".

O. Öz $(\bowtie) \cdot$ Ş. Demirkaya $\cdot$ S. Bek · E. Eroğlu

Ü. H. Ulaş · Z. Odabaş1

Neurology Department, Gülhane Military Medical Academy, Etlik, Ankara, Turkey

e-mail: oz_oguzhan@yahoo.com

\section{Introduction}

Reversible cerebral vasoconstriction syndrome (RCVS) is characterized by a sudden onset of thunderclap-like headache, with or without focal neurological deficits and seizures, most commonly in women aged 20-50 years. The onset of symptoms may be spontaneous, but a clinical association with pregnancy (postpartum angiopathy), and the intake of vasoactive drugs has been described. It is a syndrome that probably includes the previously described cases of migrainous angiitis and many cases of benign thunderclap or orgasmic headache that were not further investigated. The pathophysiology is a reversible segmental vasoconstriction of cerebral arteries that is frequently associated with focal cerebral ischemia. RCVS may be also associated with subarachnoid hemorrhage (SAH) [1-8].

About $60 \%$ of the cases of RCVS are secondary, mainly to postpartum and to the exposure to vasoactive substances. The main clinical presentation includes multiple recurrent thunderclap headaches (TCHs) over 1-4 weeks. The major complications of RCVS are localized cortical subarachnoid hemorrhages $(22 \%)$ and parenchymal strokes $(7 \%)$ that may lead to permanent sequelae [2].

Primary angiitis of the central nervous system (PACNS) is an inflammatory disease affecting the small and mediumsized leptomeningeal and intracranial vessels. Angiographic features of both RCVS and PACNS may be similar [9].

\section{Case report}

A right-handed 28-year-old woman admitted to the emergency department because of a single spontaneous severe $\mathrm{TCH}$ arising from back of neck with nausea. Her 
neurological examination was normal. Her brain computed tomography (CT) was unremarkable. Her severe headache lasted for $12 \mathrm{~h}$. She had depressive symptoms; citalopram $20 \mathrm{mg}$ and etodolak $400 \mathrm{mg}$ were prescribed. She was discharged with the diagnosis primary TCH. Her complaints were reduced by the days after this first applying but she had a moderate headache continuously. After 7 days, she had left hemiparesis. She was admitted to our neurology department and hospitalized.

She had a history of oral contraceptive medication for 5 years because of menstrual cycle disturbances (desogestrel $0,15 \mathrm{mg}+$ ethinyl estradiol $0,03 \mathrm{mg}$ ).

Clinical examination confirmed left hemiparesis, left hemihypoesthesia, left central facial paralysis. Babinski sign was positive on the left side. Blood count and biochemical analysis were normal. Brain CT and cerebrospinal fluid (CSF) examination were normal. Brain diffusionweighted magnetic resonance (MR) imaging showed acute ischemia within the right centrum semiovale, MR angiography demonstrated multiple stenotic segments both in anterior and posterior circulations particularly in the right arteria cerebri media (ACM) (Fig. 1a-c). MR venography was normal. Probabilities of SAH and cerebral venous thrombosis (CVT) were excluded. The diagnosis of ischemic cerebrovascular disease was established. Her oral contraceptive medication and citalopram discontinued. She was set on acetylsalicylic acid $300 \mathrm{mg}$ daily.

Digital subtraction angiography (DSA) showed multiple segments of narrowing in vessel calibre (Fig. 2a, c). Two probable diagnoses were PACNS and RCVS. Abdominal ultrasonography, echocardiography and carotid Doppler ultrasonography were normal.

Laboratory examinations including, lipid profile, prothrombin time, partial thromboplastin time, levels of protein $\mathrm{C}$, protein $\mathrm{S}$, anti-thrombin III, anti-phospholipid antibodies, complement, anti-neutrophil cytoplasm antibody, anti-nuclear antibody and anti-double-stranded deoxynuclear antibody anti-Ro/SSA and anti-La/SSB, ASO, homocysteine, anti-HBs, HBsAg, anti-HCV, anti-HIV $(1+2)$, VDRL were unremarkable.
Although MR angiography and DSA findings resembled PACNS, she had TCH prior to hyperacute cerebrovascular disease and did not have confusion. Because of these clinical characteristics, she was set on medication (calcium channel blocker -verapamil $240 \mathrm{mg}$ daily and acetylsalicylic acid $300 \mathrm{mg}$ daily) for probable RCVS. Follow-up MR angiography after 4 weeks and DSA after 7 weeks demonstrated improvement in alternating stenotic areas in vessels (Fig. 2b, d). Thus, diagnosis RCVS was established.

\section{Discussion}

Reversible cerebral vasoconstriction syndrome was first described by Call et al. in 1988. It is characterized by a sudden onset of thunderclap-like headache and focal neurological deficits, most commonly in women aged 20 50 years. The existing of diffuse segmental cerebral vasospasm involving the distal internal carotid, basilar, and major arteries of the circle of Willis which was fully reversible within weeks to months is the common finding in all patients [1].

Similar clinical-angiographic patterns (sudden severe headache and diffuse vasospasm) have been described in patients with a history of migraine, as well as after carotid endarterectomy, postpartum period, Guillain-Barré syndrome, sexual intercourse, unruptured intracranial aneurysm, exertion, severe hypertension, and after administration of serotonergic, sympathomimetic medications (paroxetine, sertraline, trazodone, ephedrin etc), and illicit drugs such as cocaine [10].

The headaches in RCVS are secondary headaches, symptom of this vascular disorder, that have nothing to do with migraine, which is a primary headache without underlying causal lesion. A history of migraine satisfying the International Headache Society diagnosis criteria is found in only 16-20\% of RCVS cases in prospective series $[2,11]$, a percentage that is not different from the prevalence of migraine in the general population of similar gender and age.
Fig. 1 Brain magnetic resonance imaging FLAIR (a) and diffusion-weighted (b) sequences showed acute ischemia within the right centrum semiovale. MR angiography demonstrated multiple stenotic segments both in anterior and posterior circulations particularly in the right arteria cerebri media (c)
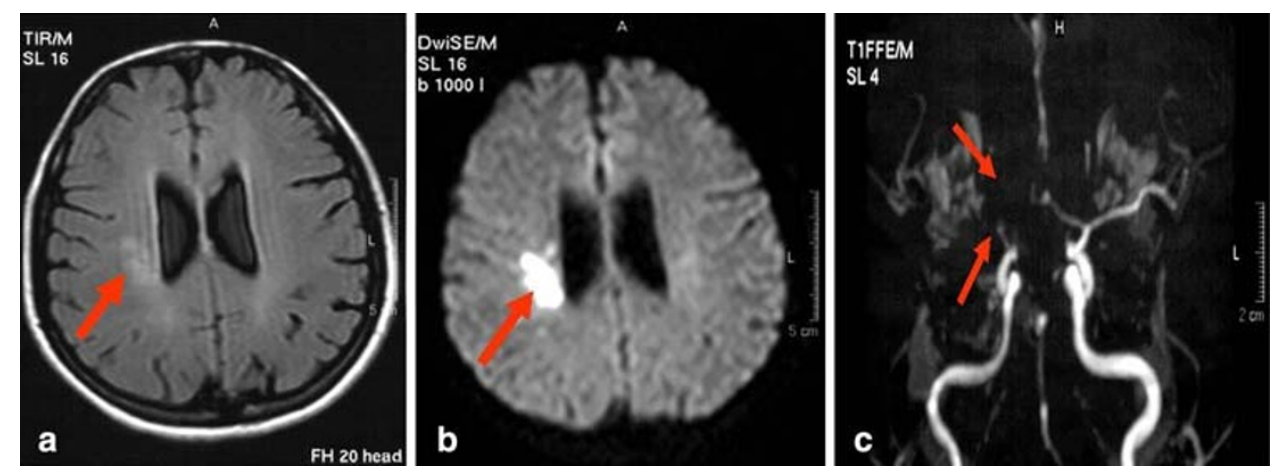
Fig. 2 Digital subtraction angiography revealed multifocal segmental vasoconstriction in anterior circulation (a) which largely resolved 7 weeks later (b). Multiple segments of narrowing in vessel calibre in basilar artery (c) were also improved after therapy (d)
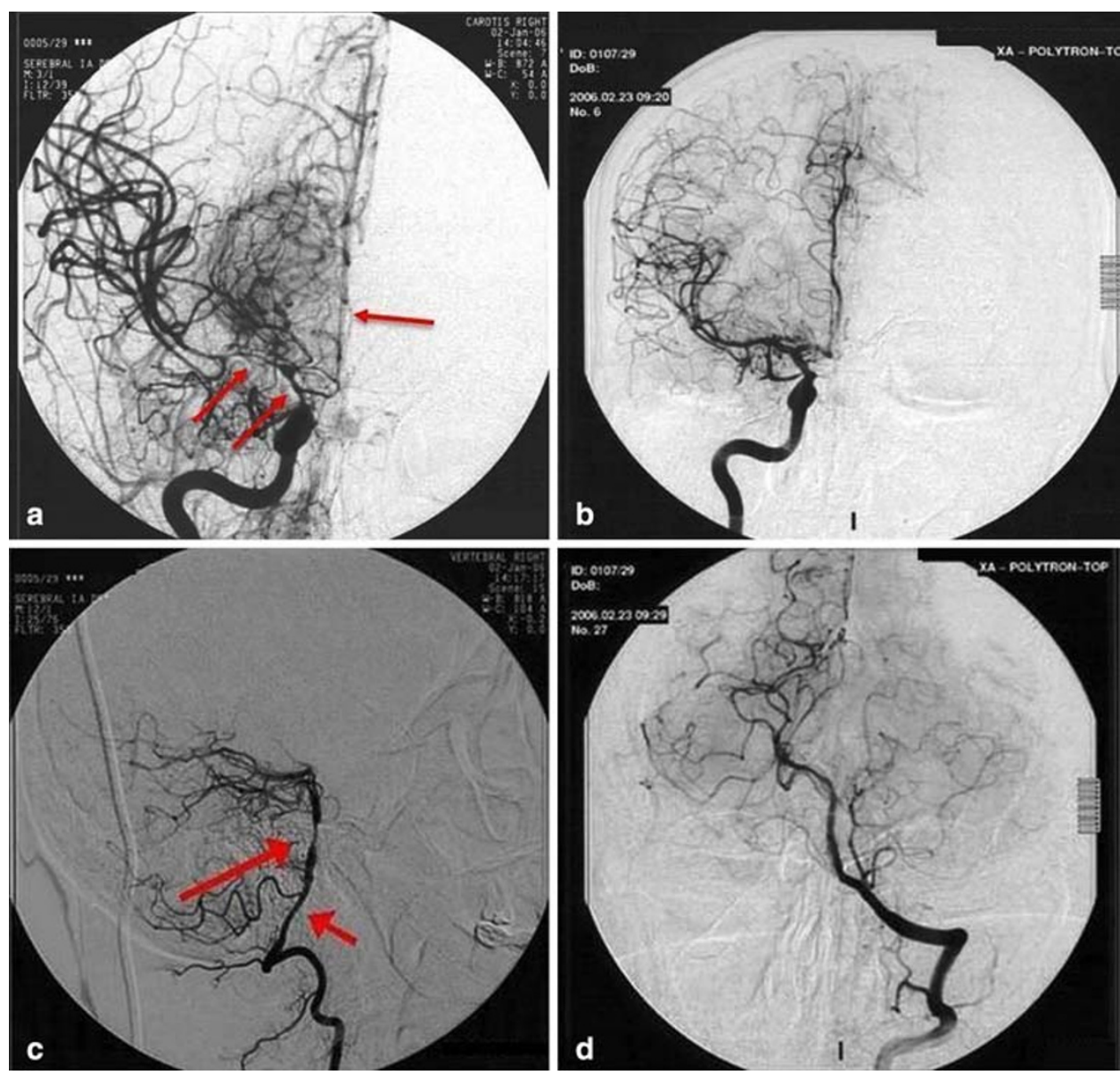

Our patient had severe headache arising from back of neck (thunderclap) with nausea, after a period of 7 days, she developed left hemiparesis. In RCVS complications occur with different time courses: cortical subarachnoid hemorrhages, intracerebral hemorrhages, seizures and posterior reversible encephalopathy syndrome are early events occurring during the first week, while ischemic events including transient ischemic attacks and cerebral infarcts occur later, during the second week. Ducros et al. [2] suggest that the underlying disturbance in the control of cerebral arterial tone first involves small distal arteries responsible for hemorrhages and then progresses towards medium and large-sized arteries responsible for ischemic events. This is exactly what happened in our case that thunderclap-like headache precedes neurological symptoms of a week.

Because of normal initial brain CT scan and normal CSF examination diagnosis of SAH and because of normal MR venography diagnosis of CVT were excluded. MR angiography demonstrated multiple stenotic segments both in anterior and posterior circulations particularly in right ACM. DSA showed multiple segments of narrowing in vessel calibre. By these findings two probable diagnoses performed, PACNS and RCVS. Because of initial clinical characteristics and normal CSF findings and because of improving clinical findings she was set on medication for probable RCVS. Follow-up MR angiography after 4 weeks and DSA after 7 weeks demonstrated improvement in vessel calibre.

The medical history of oral contraceptive medication for 5 years because of menstrual cycle disturbances and citalopram for 5 days were evaluated as important risk factors. In our case RCVS was spontaneous; unfortunately, she was treated by citalopram and had an ischemic stroke after 7 days. Selective-serotonin reuptake inhibitors (SSRIs) should be avoided in patients evaluated with the diagnosis of TCH of unknown etiology or "idiopathic thunderclap headache". Perhaps, citalopram aggravated the cerebral vasoconstriction.

It is impossible to distinguish RCVS and PACNS on the basis of angiographic features. Patients with RCVS erroneously may be treated as vasculitis, and may be exposed to long-term treatment by immunosuppressive agents with serious side effects. In the rare cases of persistent hesitation between the two diagnoses, it may be a good choice to wait a few days with a calcium channel blocker (e.g., verapamil, nimodipine): RCVS is going to stabilize and improve quickly with regression of the vasoconstriction, and on the 
contrary, arterial irregularities in PACNS are not going to improve so fast. Heavy treatment with immunosuppressants should be reserved to patients having a biopsy proven vasculitis. Consequently in these patients DSA should be repeated for definitive diagnosis.

Diagnosis and management of $\mathrm{TCH}$ contain many potential difficulties. Patients applying with TCH, particularly 20-50 years aged females should first be evaluated for common conditions, such as SAH and also RCVS must be considered. Clinicians should consider the imaging of cerebral arteries, even if CT scan and lumbar puncture are normal in TCH. Recognition of RCVS may be life-saving, potential precipitating factors such as postpartum period and vasoactive substances especially SSRIs and triggers (sexual intercourse, defecation, sudden emotion, physical exertion, urination without effort, cough, sneezing, bathing or showering and sudden head movement) should also be known and avoided.

Conflict of interest None.

\section{References}

1. Call GK, Fleming ML, Sealfon S, Levine H, Kistler JP, Fisher CM (1988) Reversible cerebral segmental vasoconstriction. Stroke 19:1159-1170

2. Ducros A, Boukobza M, Porcher R, Sarov M, Valade D, Bousser MG (2007) The clinical and radiological spectrum of reversible cerebral vasoconstriction syndrome. A prospective series of 67 patients. Brain 130(Pt 12):3091-3101

3. Singhal AB, Caviness VS, Begleiter AF, Mark EJ, Rordorf G, Koroshetz WJ (2002) Cerebral vasoconstriction and stroke after use of serotonergic drugs. Neurology 58:130-133

4. Koopman K, Teune LK, Ter Laan M, Uyttenboogaart M, Vroomen PC, De Keyser J, Luijckx GJ (2008) An often unrecognized cause of thunderclap headache: reversible cerebral vasoconstriction syndrome. J Headache Pain 9(6):389-391

5. Reneman L, Habraken JB, Majoie CB, Booij J, den Heeten GJ (2000) MDMA ('Ecstasy') and its association with cerebrovascular accidents: preliminary findings. Am J Neuroradiol 21:1001-1007

6. Neudecker S, Stock K, Krasnianski M (2006) Call-Fleming postpartum angiopathy in the puerperium: a reversible cerebral vasoconstriction syndrome. Obstet Gynecol 107(2 Pt 2):446-449 (Comment in: Obstet Gynecol. 2006 Feb;107(2 Pt 2):437-438)

7. Moustafa RR, Allen CM, Baron JC (2008) Call-Fleming syndrome associated with subarachnoid haemorrhage: three new cases. J Neurol Neurosurg Psychiatry 79(5):602-605 [Epub 2007 Dec 12]

8. Keyrouz S, Dhar R, Axelrod Y (2008) Call-Fleming syndrome and orgasmic cephalgia. Headache 48(6):967-971

9. Koopman K, Uyttenboogaart M, Luijckx GJ, De Keyser J, Vroomen PC (2007) Pitfalls in the diagnosis of reversible cerebral vasoconstriction syndrome and primary angiitis of the central nervous system. Eur J Neurol 14:1085-1087

10. Dodick DW (2003) Reversible segmental cerebral vasoconstriction (Call-Fleming syndrome): the role of calcium antagonists. Cephalalgia 23(3):163-165 (Comment on: Cephalalgia 2003 Apr; 23(3):218-222)

11. Chen SP, Fuh JL, Lirng JF, Chang FC, Wang SJ (2006) Recurrent primary thunderclap headache and benign CNS angiopathy: spectra of the same disorder? Neurology 67(12):2164-2169 